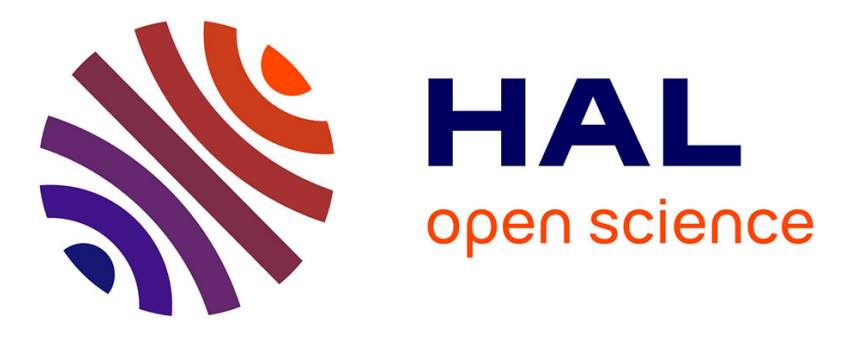

\title{
Polar Fe(IO3)3 nanocrystals as local probes for nonlinear microscopy
}

\author{
L. Bonacina, Y. Mugnier, F. Courvoisier, R. Le Dantec, J. Extermann, \\ Yannick Lambert, V. Boutou, C. Galez, J.-P. Wolf
}

\section{- To cite this version:}

L. Bonacina, Y. Mugnier, F. Courvoisier, R. Le Dantec, J. Extermann, et al.. Polar Fe(IO3)3 nanocrystals as local probes for nonlinear microscopy. Applied Physics B - Laser and Optics, 2007, 87 (3), pp.399-403. 10.1007/s00340-007-2612-z . hal-00494021

\section{HAL Id: hal-00494021 https://hal.science/hal-00494021}

Submitted on 6 May 2021

HAL is a multi-disciplinary open access archive for the deposit and dissemination of scientific research documents, whether they are published or not. The documents may come from teaching and research institutions in France or abroad, or from public or private research centers.
L'archive ouverte pluridisciplinaire HAL, est destinée au dépôt et à la diffusion de documents scientifiques de niveau recherche, publiés ou non, émanant des établissements d'enseignement et de recherche français ou étrangers, des laboratoires publics ou privés.

\section{(c)(1)}

Distributed under a Creative Commons Attribution| 4.0 International License 


\title{
Polar $\mathrm{Fe}\left(\mathrm{IO}_{3}\right)_{3}$ nanocrystals as local probes for nonlinear microscopy
}

\author{
L. Bonacina ${ }^{1}$, Y. Mugnier ${ }^{2}$, F. Courvoisier ${ }^{1}$, R. Le Dantec ${ }^{2}$, J. Extermann ${ }^{1}$, Y. Lambert ${ }^{2}$, V. Boutou ${ }^{3}$, C. Galez ${ }^{2}$, J.-P. Wolf ${ }^{1}$
}

${ }^{1}$ GAP-Biophotonics, Université de Genève, 20 rue de l'Ecole de Médecine, 1211 Geneva 4, Switzerland

2 SYMME, Polytech'Savoie, Université de Savoie, BP 80439, 7944 Annecy Le Vieux, France

${ }^{3}$ LASIM, Université Claude Bernard Lyon 1, 43 bd du 11 Novembre 1918, 69622 Villeurbanne Cedex, France

\begin{abstract}
We have investigated nanocrystals of $\mathrm{Fe}\left(\mathrm{IO}_{3}\right)_{3}$ by polarization-sensitive second harmonic generation ( $\mathrm{SHG}) \mathrm{mi}$ croscopy. As the nonlinear optical properties of this material were only poorly characterized, we have first determined the relative values of the elements of its second-order susceptibility tensor, by the global fitting of the polarization-resolved SHG response of an ensemble of nanocrystals. This information allows one to optically retrieve the orientation of individual particles in the sample. The high SHG efficiency measured for nanocrystals of $\mathrm{Fe}\left(\mathrm{IO}_{3}\right)_{3}$ and their polar nature could make them very attractive for nonlinear microscopy of biological samples.
\end{abstract}

\section{$1 \quad$ Introduction}

Nonlinear optics in nanoparticles recently emerged as a very active field of research. In particular, second harmonic generation (SHG) was investigated both theoretically [1-4] and experimentally on metal- [5,6], semiconductor- $[7,8]$, and organic nanoparticles $[9,10]$. Although many studies were dedicated to nanostructures or to ensembles of particles, SHG imaging of single nanorods and single organic nanocrystals was only recently demonstrated using laser scanning microscopy [10] and SNOM [8,9]. A major application of SHG microscopy is imaging of biological samples [11]. Fluorescent dyes (cyanines, GFP, YFP, CFP, etc.) are commonly used as markers. However these molecules suffer from a major drawback: fast photo-degradation. Bleaching occurs already after the emission of $10^{6}-10^{8}$ photons in organic dyes [12] and $<10^{5}$ photons in autofluorescent proteins [13]. Fluorescing semiconductor quantum dots provide much higher photostability, but are known to suffer from blinking (strong fluorescence intensity variations over unpredictable timescales) $[14,15]$. Provided that SHG conversion efficiency is large enough, SHG appears as an attractive alternative to fluorescence, as no bleaching nor blinking effects (no absorption) are expected $[16,17]$. The absence of phasematching requirements in nanoparticles allows a large tun-

Fax: +330450 096649, E-mail: yannick.mugnier@univ-savoie.fr ability of the excitation and provides the possibility to double the full frequency spectrum of broad sources. This capability could be used for identifying the multiple scattering paths of the various spectral components of the incoming light improving depth resolution in tissues [18].

In order to obtain high SHG conversion efficiency, noncentrosymmetric inorganic nanocrystals are of particular interest. We previously showed that iron iodate $\left(\mathrm{Fe}\left(\mathrm{IO}_{3}\right)_{3}\right)$ can be grown as nanocrystals with a simple and inexpensive process, and is an excellent candidate for nanodoublers. Moreover $\mathrm{Fe}\left(\mathrm{IO}_{3}\right)_{3}$ has low-chemical reactivity, and it is stable in aqueous solution in a wide $\mathrm{pH}$ and temperature range (up to $400{ }^{\circ} \mathrm{C}$ ) [19], contrary to SHG active organic crystals [20]. It could also be potentially used as a nanoprobe of the local electric field by taking advantage of its polar structure.

We used polarization-sensitive SHG microscopy of $\mathrm{Fe}\left(\mathrm{IO}_{3}\right)_{3}$ nanocrystals associated to a robust fitting procedure, in order to achieve the following: 1) retrieve quantitative information on the elements of the previously unknown secondorder susceptibility tensor of this material, and 2) determine the spatial orientation of each nanodoubler.

\section{Experimental \\ $2.1 \quad \mathrm{Fe}\left(\mathrm{IO}_{3}\right)_{3}$ sample}

Iron iodate was synthesized in the form of a powder of agglomerated nanocrystals by a co-precipitation method. Although dispersion of nanocrystals is crucial to obtain a homogeneous suspension suited for biomedical imaging, coprecipitation in aqueous solution is a cost-effective way to elaborate nanomaterials with specific properties. Recent studies have shown that the size of nanocrystals can be adjusted according to the experimental parameters of the synthesis [21]. Co-precipitation in aqueous solution of $\mathrm{Fe}\left(\mathrm{IO}_{3}\right)_{3}$ powder is obtained from iron nitrate $\left(\mathrm{Fe}\left(\mathrm{NO}_{3}\right)_{3} \cdot 9 \mathrm{H}_{2} \mathrm{O}\right.$, Aldrich, 98\%) and iodic acid ( $\mathrm{HIO}_{3}$, Aldrich, 99.5\%). The reactants are separately dissolved in distilled water, then the iron nitrate solution is progressively poured into the iodic acid solution while vigorous stirring is maintained. After being heated at $80{ }^{\circ} \mathrm{C}$ for 2 days, the suspension is filtered $(30 \mu \mathrm{m})$ and dried in an air flow at room temperature [19]. The powder is successively diluted in water, and a drop of the filtered $(0.22 \mu \mathrm{m})$ solution is dried on a graphite substrate or a standard microscope glass slide. 


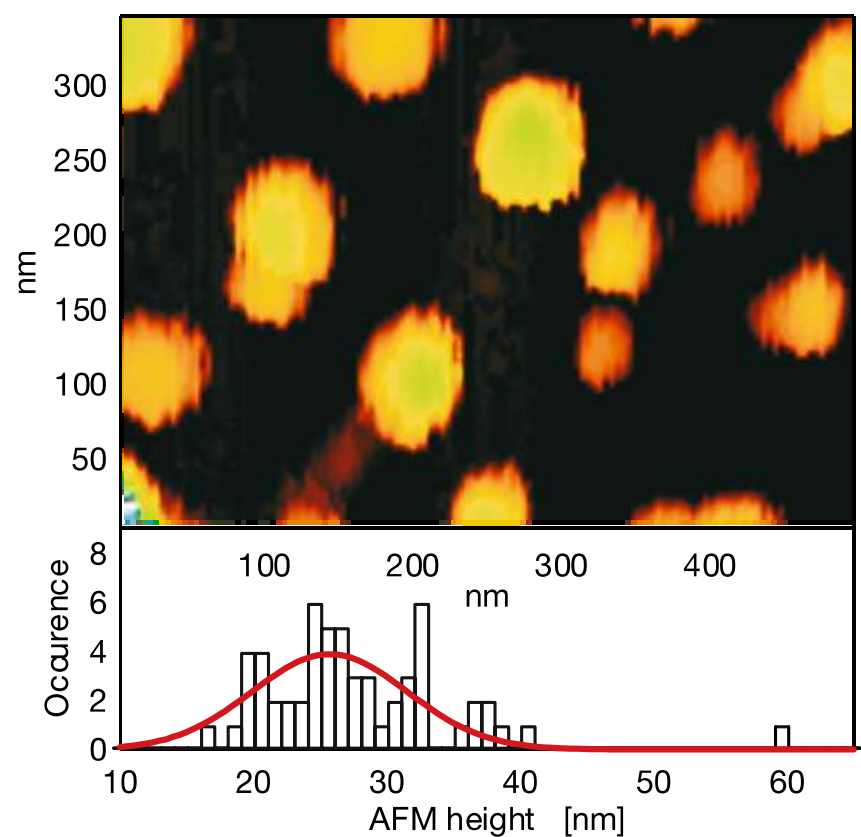

FIGURE 1 IC-AFM image of $\mathrm{Fe}\left(\mathrm{IO}_{3}\right)_{3}$ nanocrystals deposited on a graphite substrate. The histogram in the lower panel illustrates the size distribution of 60 nanocrystals, and it is fitted by a Gaussian centered at $27.7 \mathrm{~nm}$ of FWHM $13.7 \mathrm{~nm}$

$\mathrm{X}$-ray powder diffraction indicates that the mean particle size is 20-40 nm [19], as confirmed (Fig. 1) by intermittentcontact atomic force microscopy (IC-AFM). These data were collected for nanocrystals dispersed on a graphite substrate. For the SHG experiments we had to work with standard microscopic glass slides, where we observed a stronger tendency of $\mathrm{Fe}\left(\mathrm{IO}_{3}\right)_{3}$ nanoparticles to aggregate, as illustrated by the size distribution in Fig. 2 centered around $80 \mathrm{~nm}$.

\section{2}

\section{Optical set-up}

The SHG images were acquired using an inverted laser scanning microscope (Nikon TE300), equipped with a 1.3 NA $100 \times$ oil immersion objective. The position of the sample was controlled by an $X Y Z$ piezo-scanner with a resolution of $2 \mathrm{~nm}$ on each axis. The excitation source was a mode locked Ti:sapphire oscillator (Kapteyn-Murnane Labs), providing $20 \mathrm{fs}$ pulses at $100 \mathrm{MHz}$ repetition rate. The incident light polarization was selected by a rotating half wave plate, while SHG signal was collected in the backward direction by the same objective. A combination of a BG40 Schott filter, and a bandpass filter ( $40 \mathrm{~nm}$ bandwidth at $400 \mathrm{~nm}$ ) assured an efficient rejection of the $800 \mathrm{~nm}$ scattering. After being analyzed by a Glan-Taylor polarization cube, the signal was measured by a photomultiplier tube and processed by a lock-in amplifier.

\section{3}

\section{Results}

The sample was first characterized (imaging and sizing) by AFM, and labelled to allow a straight comparison with the results obtained by SHG microscopy (Fig. 2). Apart from its intrinsic lower spatial resolution $(\sim 500 \mathrm{~nm})$, the latter technique was able to retrieve the positions of almost all the nanocrystals dispersed on the microscope slide. In scanning

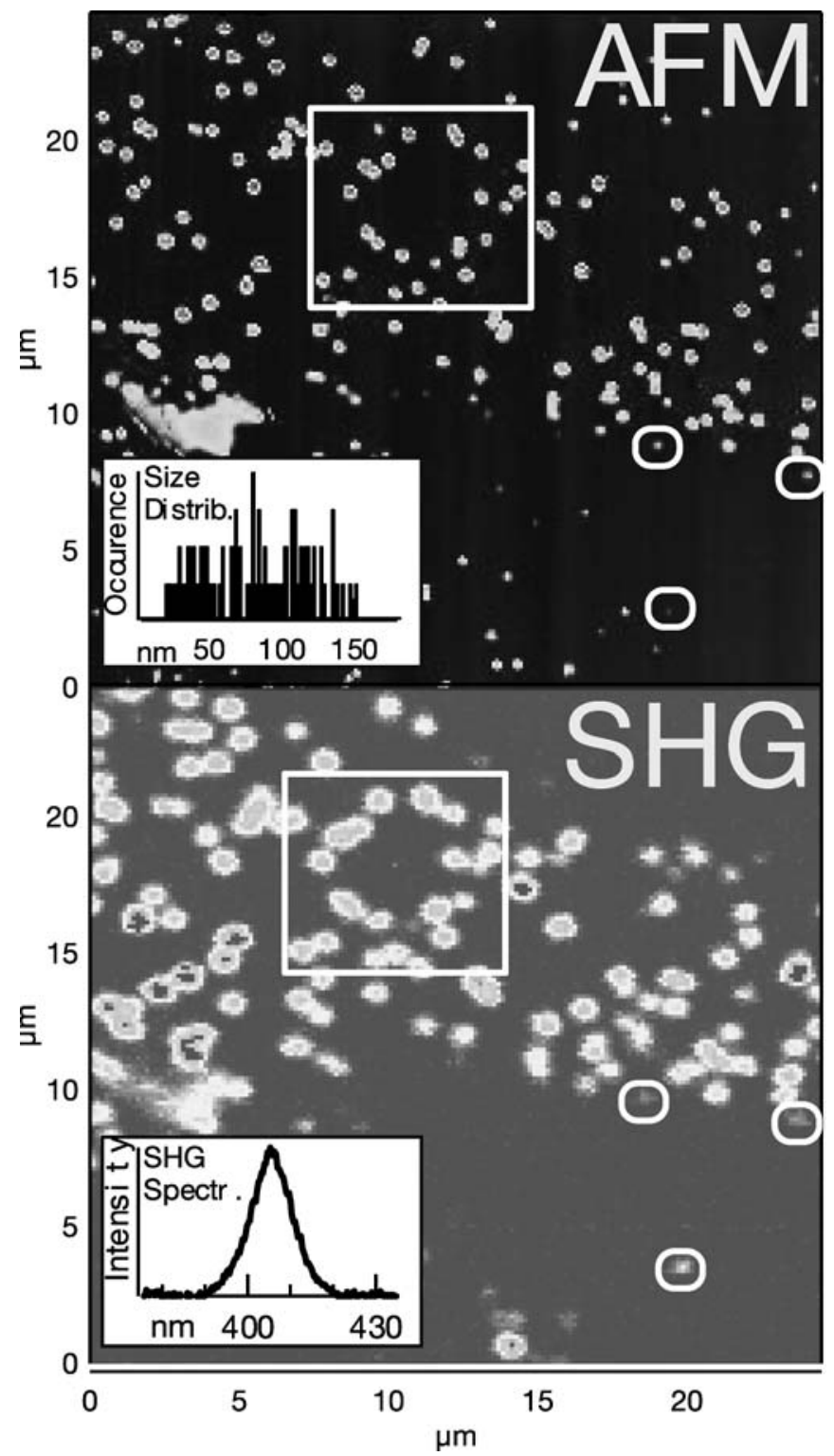

FIGURE 2 Upper panel: IC-AFM image of $\mathrm{Fe}\left(\mathrm{IO}_{3}\right)_{3}$ nanocrystals deposited on a glass slide. Inset: distribution of AFM heights of the sample. Lower panel: SHG image of the same sample region. The SHG image was obtained by averaging two scans associated with perpendicular polarizations of the excitation laser and no polarization selection at the detector. The three open circles highlight nanocrystals with measured height at $40 \mathrm{~nm}$ and AFM width below $350 \mathrm{~nm}$. The open square is a guideline to the eye for an easier comparison of the two images. Inset: SHG spectrum emitted by a single nanocrystal

mode we were able to identify the SHG signal of individual crystals as small as $50 \mathrm{~nm}$ height and $150 \mathrm{~nm}$ lateral size. The white circles in the figure highlight three nanocrystals of $40 \mathrm{~nm}$ height and AFM width below $350 \mathrm{~nm}$ to prove the spatial sensitivity of the technique. The inset in the lower panel shows the SHG spectrum emitted by a single nanocrystal. The spectral full width half maximum of the second harmonic emission is $12 \mathrm{~nm}$, which corresponds to doubling the whole fundamental laser spectrum $(\sim 50 \mathrm{~nm}$ at $810 \mathrm{~nm})$.

To detect the individual orientation in space of each nanocrystal the laser was first focused onto selected crystals, previously characterized in size by AFM. During this oper- 
ation, the SHG signal was carefully maximized by adjusting the microscope piezo-scanner displacements. The polarization response was successively acquired measuring the SHG signal as a function of the polarization angle of the incident light, $\gamma$, and of the detection polarization, setting the analyzer along the $X$ or $Y$ direction (see definitions in Fig. 3). The data presented in Fig. 4 were acquired integrating $100 \mathrm{~ms}$ on the lock-in with a post-averaging over 10 points.

The experimental SHG polarization response was fitted according to the following assumptions. The fundamental beam is supposed at normal incidence, with only two in-plane components [10], $E_{x}^{\omega}=E^{\omega} \cos (\gamma)$ and $E_{y}^{\omega}=E^{\omega} \sin (\gamma)$. The collected SHG intensity in the $X$ direction $I_{X}$ (resp. $I_{Y}$ ) is then assumed to be proportional to $P_{X}^{2}$ (resp. $P_{Y}^{2}$ ), where $\boldsymbol{P}$ is the nonlinear polarization given by:

$P_{i}^{2 \omega}=\varepsilon_{0} \sum_{j k} \chi_{i j k} E_{j}^{\omega} E_{k}^{\omega}$

The crystal nonlinear susceptibility tensor, $\chi_{i j k}$, is given here in the laboratory frame and can be derived from the $\chi_{\bar{i} \bar{j} \bar{k}}$ tensor expressed in the crystal frame by:

$\chi_{i j k}=\sum_{\bar{i} \bar{j} \bar{k}} \chi_{\bar{i} \bar{j} \bar{k}} S_{i \bar{i}} S_{j \bar{j}} S_{k \bar{k}}$

where $S_{i \bar{i}}$ are the components of the rotation matrix between the laboratory and crystal axes depending on the Euler angles $\varphi, \theta$ and $\psi$.

Since the basic physical properties of $\mathrm{Fe}\left(\mathrm{IO}_{3}\right)_{3}$ are still unknown, as the bulk counterpart is not easily grown [22, 23], we first tested the procedure on a bulk crystal of well known nonlinear optical properties. As shown in Fig. 4a, the SHG polarization response of bulk $\mathrm{LiNbO}_{3}$ was found in excellent agreement with the theoretical curve obtained using the literature values for the $\chi_{i j k}$ tensor [24], and the experimentally measured Euler angles $\left(\varphi=88^{\circ} \mathrm{C}, \theta=90^{\circ} \mathrm{C}, \psi=\right.$ $90^{\circ} \mathrm{C}$ ). Models more sophisticated than the present one have been proposed to describe the response of multiphoton microscopy [11,25-27], accounting for the effect of a high NA objective in the collection of the backward propagating radiation and in the polarization dependent response. To verify the

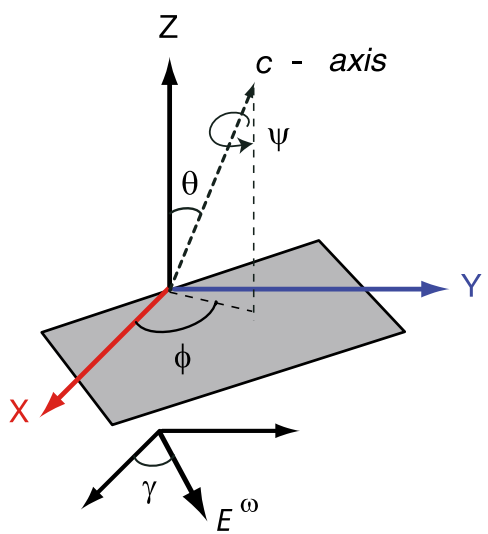

FIGURE 3 Definition of the angles. The $c$-axis of the crystal is expressed in the laboratory frame $X, Y, Z$ by the Euler angles $\varphi, \theta$ and $\psi$, whereas the angle $\gamma$ denotes the polarization of incident laser light on the sample plane

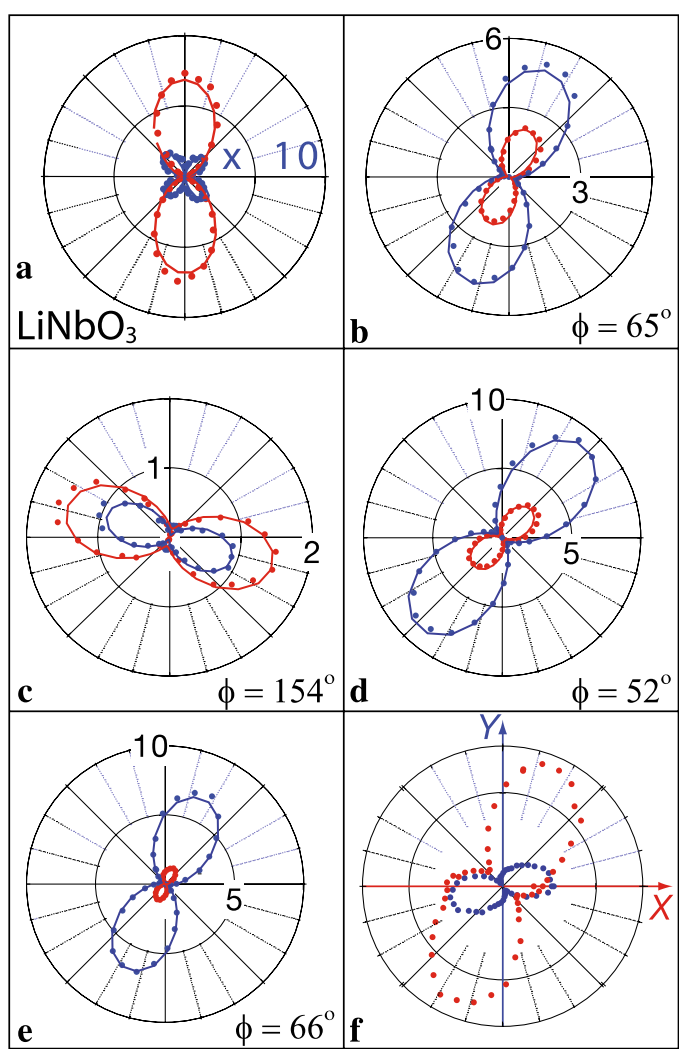

FIGURE 4 Polarization response of SHG emission analyzed along the $X$ (blue) and $Y$ (red) direction. The dots are experimental points, the solid lines the best fit according to the model described in the text. (a) Bulk $\mathrm{LiNbO}_{3}$ plate. $I_{X}$ is multiplied by a factor 10 for easier inspection. (b-e) Examples taken from an ensemble of 12 nanocrystals simultaneously fitted. (f) Typical response from a non-monocrystalline aggregate

accuracy of our simplified approach which neglects these aspects, we have simulated the effect of wide angle collection of our 1.3 NA objective and included in the calculation the dichroism and ellipticity of our apparatus. Under certain crystals orientations, as shown in Fig. 4a, we did not observe any difference between the measurements and both the complete and the simplified model. However, as discussed in [25], the axial components of the electric field can play a role in the polarization response. For example, we could remark some discrepancies between the experimental response of bulk lithium niobate ( $Z$-cut) and the predictions of the simplified model, which can be reduced applying the complete treatment. These effects were not retained in the fitting procedure described here exclusively for computational reason. In fact, we needed to dispose of an analytical compact expression for $I_{X}$ and $I_{Y}$ that could not be derived in the framework of the complete model.

Iron iodate belongs to the symmetry class 6 , and thus only 4 components of the nonlinear tensor are expected to be non-zero: $\chi_{z z z}, \chi_{z x x}=\chi_{z y y}, \chi_{x x z}=\chi_{x z x}=\chi_{y y z}=\chi_{y z y}, \chi_{x y z}=$ $\chi_{x z y}=-\chi_{y z x}=-\chi_{y x z}$. Given that the absolute values of these nonlinear coefficients are not known, the symmetry of the nonlinear tensor represents the only fixed input parameter that can be employed in the model. As the SHG intensity in the $X$ or $Y$ direction of the $n$-labelled crystal can be computed as $I_{X}^{n}=K f_{x}\left(\varphi^{n}, \theta^{n}, \chi_{i j k}\right) g\left(v^{n}\right)$ and $I_{Y}^{n}=K f_{y}\left(\varphi^{n}, \theta^{n}, \chi_{i j k}\right) g\left(v^{n}\right)$, 7 free parameters remain to be adjusted for the fit: the 4 
non-zero $\chi_{i j k}$ elements, the Euler angles $\varphi^{n}$ and $\theta^{n}$, and the function $g\left(v^{n}\right)$, which relates the crystal size $v^{n}$ to the SHG intensity. The experimental factor $K$ accounts for the incident laser intensity, the collection and the detection efficiency. Note that, according to the crystal symmetry $\left(P 6_{3}\right)$, the angle $\psi$ has no influence on the nonlinear optical response. Siltanen et al. [28] have shown that it is possible to uniquely determine the values of six parameters by fitting the polarization responses $I_{X}$ and $I_{Y}$ of a sample. The system is, thus, under-determined when fitting exclusively the response of a single nanocrystal. On the other hand, when fitting simultaneously the polarized SHG emission of several nanocrystals, the system is over-determined, and the relative values of the nonlinear susceptibility tensor, as well as the orientation of each nanoparticle, become accessible. Consequently, we adopted a global fitting procedure, and we fitted simultaneously the SHG data collected from 12 nanocrystals considering $\chi_{i j k}$ as a global parameter. ${ }^{1}$ The experimental curves in Fig. 4 (panels $b$ to e) are very well reproduced using the form of $\chi_{i j k}$ tensor associated to the $\mathrm{Fe}\left(\mathrm{IO}_{3}\right)_{3}$ symmetry. Although the intensities of the signals span more than one order of magnitude, the fitting procedure is very robust in indicating the in-plane orientation of each crystal, $\varphi^{n}$, which connotes unambiguously the shape and the relative intensities of $I_{X}^{n}$ and $I_{Y}^{n}$. Note that even small differences of the $\varphi^{n}$ angles lead to responses that can be distinguished by the experiment.

The absolute intensities of $I_{X}$ and $I_{Y}$ are determined conjointly by the off-plane angle $\theta^{n}$, the elements of the $\chi_{i j k}$ tensor, and the value assumed by the volume-function $g\left(v^{n}\right)$. A lack of modelling concerning the exact expression for $g\left(v^{n}\right)$, i.e., the correspondence between the nanocrystal size and the backward propagating SHG emission, is at the origin of the spread in the values of $\chi_{i j k} / \chi_{z z z}$ obtained for different fits and summarized in Table 1 . All fits coincide in indicating that the element $\chi_{z z z}$ is by far the dominant one, $\chi_{z x x} \geq \chi_{x y z}$, and that $\chi_{x x z}$ is almost negligible. The values obtained for the off-plane angles $\theta^{n}$, directly reflect the indeterminacy shown for $\chi_{i j k}$. As already pointed out, the results retrieved for the angles $\varphi^{n}$ remain the same in all the fits performed.

The SHG signals emitted by larger structures, typically of $\geq 0.8 \mu \mathrm{m}$ AFM lateral size, cannot be satisfactorily fitted using the assumptions described above. Very likely, these particles are polycrystalline aggregates made up by several crystal structures oriented in different directions, and their polarization response results in a superposition of signals generated by the different domains (plot in panel f). Therefore, once the relative values of $\chi_{i j k}$ are known, the polarization response allows a rapid in situ inspection of the local crystalline order, and allows one to tell apart monocrystalline structures from polycrystalline aggregates as recently observed for organic nanocrystals [10].

The quantitative determination of $\chi_{i j k}$ of $\mathrm{Fe}\left(\mathrm{IO}_{3}\right)_{3}$ is currently under progress, by comparison with the responses of several different bulk materials, and by adopting an expression for $g\left(v^{n}\right)$ accounting for tight focused excitation, and coherent superposition effects as a function of sam-

\footnotetext{
${ }^{1}$ The same procedure applied to different ensembles/numbers of particles yielded similar results.
}

\begin{tabular}{ccc}
\hline$\left|\chi_{z x x} / \chi_{z z z}\right|$ & $\left|\chi_{x y z} / \chi_{z z z}\right|$ & $\left|\chi_{x x z} / \chi_{z z z}\right|$ \\
\hline $0.03-0.2$ & $0.03-0.1$ & $<10^{-2}$
\end{tabular}

TABLE 1 Relative values of the non-zero elements of the $\chi$-tensor of $\mathrm{Fe}\left(\mathrm{IO}_{3}\right)_{3}$ retrieved from the global fitting of 12 nanocrystals SHG polarization response

ple size $[2,25,26]$. However, to give an estimate and allow a comparison with other recent works, we applied the procedure outlined by Delahaye et al. [29] to evaluate $\chi_{z z z}$ using as reference signal the response of a bulk $\mathrm{LiNbO}_{3}$ crystal. Within this framework $g\left(v^{n}\right)=\left(v^{n}\right)^{2}$. Adopting for the calculation the response of $\mathrm{a} \sim 0.6 \mu \mathrm{m}$ particle, we estimated $\chi_{z z z} \simeq 18 \mathrm{pm} / \mathrm{V}$, in agreement with the order of magnitude deduced from powder measurements [23]. Nevertheless, this value should be taken with caution, because the approximations of this treatment lead to a strong dependency of its outcome on the size of the nanocrystal. The complementarity of AFM and SHG microscopy in our experiment has the advantage of exposing immediately the limitations of stating such a simplified correspondence between the response of a massive crystal and that of a nano-object.

4

\section{Conclusions}

We have performed a detailed analysis of the polarization response of nanocrystals of $\mathrm{Fe}\left(\mathrm{IO}_{3}\right)_{3}$, which can be easily synthesized by an inexpensive chemical method. Due to the difficulty of growing bulk crystals of this material, its nonlinear properties were previously only partially known. We demonstrated that $\mathrm{Fe}\left(\mathrm{IO}_{3}\right)_{3}$ is an efficient frequency doubler, and this property, together with its remarkable stability in aqueous solution even under extreme $\mathrm{pH}$ and temperature conditions, makes of it a very attractive probe for SHG microscopy. Moreover, the possibility to interrogate optically the orientation of each nanocrystal opens the way to a large number of applications. Contrary to similar studies where SHG active particles were embedded in host media (sol-gel matrices, polymers, inclusion compounds), the present measurements were taken directly on nanocrystal isolated on a substrate to prove their aptness as markers for microscopy.

\section{REFERENCES}

1 J.I. Dadap, J. Shan, K.B. Eisenthal, T.F. Heinz, Phys. Rev. Lett. 83, 4045 (1999)

2 J. Mertz, L. Moreaux, Opt. Commun. 196, 325 (2001)

3 Y. Pavlyukh, W. Hubner, Phys. Rev. B 70, 245434 (2004)

4 K. Nappa, I. Russier-Antoine, E. Benichou, C. Jonin, P.F. Brevet, Chem. Phys. Lett. 415, 246 (2005)

5 J. Nappa, G. Revillod, I. Russier-Antoine, E. Benichou, C. Jonin, P.F. Brevet, Phys. Rev. B 71, 165407 (2005)

6 M.L. Sandrock, C.D. Pibel, F.M. Geiger, C.A. Foss, J. Phys. Chem. B 103, 2668 (1999)

7 S.W. Chan, R. Barille, J.M. Nunzi, K.H. Tam, Y.H. Leung, W.K. Chan, A.B. Djurisic, Appl. Phys. B 84, 351 (2006)

8 J.C. Johnson, H. Yan, R.D. Schaller, P.B. Petersen, P. Yang, R.J. Saykally, Nano Lett. 2, 279 (2002)

9 Y.Z. Shen, P. Markowicz, J. Winiarz, J. Swiatkiewicz, P.N. Prasad, Opt. Lett. 26, 725 (2001) 
10 S. Brasselet, V. Le Floc'h, F. Treussart, J.F. Roch, J. Zyss, E. BotzungAppert, A. Ibanez, Phys. Rev. Lett. 92, 207401 (2004)

11 L. Moreaux, O. Sandre, J. Mertz, J. Opt. Soc. Am. B 17, 1685 (2000)

12 L. Cognet, Lasers et Technologies Femtosecondes, 1st edn. (Publications de l'Université, Saint-Etienne, 2005)

13 G.S. Harms, L. Cognet, P.H.M. Lommerse, G.A. Blab, T. Schmidt, Biophys. J. 80, 2396 (2001)

14 M. Nirmal, B.O. Dabbousi, M.G. Bawendi, J.J. Macklin, J.K. Trautman, T.D. Harris, L.E. Brus, Nature 383, 802 (1996)

15 X. Brokmann, J.P. Hermier, G. Messin, P. Desbiolles, J.P. Bouchaud, M. Dahan, Phys. Rev. Lett. 90, 120601 (2003)

16 R.M. Williams, W.R. Zipfel, W.W. Webb, Curr. Opin. Chem. Biol. 5, 603 (2001)

17 J. Mertz, Curr. Opin. Neurobiol. 14, 610 (2004)

18 J.M. Dela Cruz, I. Pastirk, M. Comstock, V.V. Lozovoy, M. Dantus, Proc. Nat. Acad. Sci. USA 101, 16996 (2004)

19 C. Galez, Y. Mugnier, J. Bouillot, Y. Lambert, R. Le Dantec, J. Alloys Compd. 416, 261 (2006)
20 K. Komorowska, S. Brasselet, G. Dutier, I. Ledoux, J. Zyss, L. Poulsen, M. Jazdzyk, H. Egelhaaf, J. Gierschner, M. Hanack, Chem. Phys. 318, $12(2005)$

21 J.P. Jolivet, C. Froidefond, A. Pottier, C. Chaneac, S. Cassaignon, E. Tronc, P. Euzen, J. Mater. Chem. 14, 3281 (2004)

22 K. Nassau, J.W. Shiever, B.E. Prescott, J. Solid State Chem. 7, 186 (1973)

23 S.C. Abrahams, R.C. Sherwood, J.L. Bernstein, K. Nassau, J. Solid State Chem. 7, 205 (1973)

24 R.L. Sutherland, Handbook of Nonlinear Optics, 2nd edn. (Marcel Dekker, London, 2003)

25 E.Y.S. Yew, C.J.R. Sheppard, Opt. Express 14, 1167 (2006)

26 J.X. Cheng, X.S. Xie, J. Opt. Soc. Am. B 19, 1604 (2002)

27 W. Débarre, D. Supatto, E. Beaurepaire, Opt. Lett. 30, 2134 (2005)

28 M. Siltanen, S. Cattaneo, E. Vuorimaa, H. Lemmetyinen, T.J. Katz, K.E.S. Phillips, M. Kauranen, J. Chem. Phys. 121, 1 (2004)

29 E. Delahaye, N. Tancrez, T. Yi, I. Ledoux, J. Zyss, S. Brasselet, R. Clément, Chem. Phys. Lett. 429, 533 (2006) 